\title{
Exosomes Derived from Mouse Adipose-Derived Mesenchymal Stem Cells Alleviate Benzalkonium Chloride-Induced Mouse Dry Eye Model via Inhibiting NLRP3 Inflammasome
}

\author{
Guifang Wang $^{\mathrm{a}}$ Honghui $\mathrm{Li}^{\mathrm{b}}$ Hongmei Long ${ }^{\mathrm{c}}$ Xileyuan Gong ${ }^{\mathrm{a}}$ Shufang $\mathrm{Hu}^{\mathrm{a}}$ \\ Can Gong ${ }^{\text {a }}$ \\ ${ }^{a}$ Department of Ophthalmology, Loudi Central Hospital, Loudi, China; ${ }^{b}$ Department of Traditional Chinese \\ Medicine, Loudi Central Hospital, Loudi, China; 'Department of Endocrine, Loudi Central Hospital, Loudi, China
}

\section{Keywords}

Mouse adipose-derived mesenchymal stem cell-derived exosomes · NLR family pyrin domain-containing 3; NLRP3 inflammasome · Dry eye · Inflammation - Ocular surface

\begin{abstract}
Purpose: The objective of the study was to investigate efficacy and mechanisms of mouse adipose-derived mesenchymal stem cell-derived exosomes (mADSC-Exos) in the benzalkonium chloride (BAC)-induced mouse dry eye model. Methods: Exosomes in the mADSC culture supernatant were isolated by ultracentrifugation. Western blotting, nanoparticle tracking analysis, and transmission electron microscopy were used to characterize mADSC-Exos. An experimental mouse model of dry eye was established by instillation of $0.2 \%$ BAC. mADSC-Exos were administered following BAC treatment. The positive control group was treated with commercial eye drops ( $0.1 \%$ pranoprofen). Corneal fluorescein staining, tear secretion, and tear film break-up time (BUT) were evaluated, and histologic analysis of the cornea and conjunctiva was performed by hematoxylin and eosin and
\end{abstract}

periodic acid-Schiff staining. Apoptosis in the corneal epithelium was detected with the terminal deoxynucleotidyl transferase dUTP nick-end labeling assay and by Western blotting. Levels of pro-inflammatory cytokines in the cornea and conjunctiva were evaluated by flow cytometry, and mRNA and protein levels of NLR family pyrin domain-containing 3 (NLRP3) pathway components were assessed by quantitative real-time PCR and Western blotting, respectively. Results: mADSC-Exos were characterized as vesicles with a bilayer membrane. The particle size distribution peak was at $134 \mathrm{~nm}$. mADSC-Exos specifically expressed cluster of differentiation (CD)9, CD63, and CD81. mADSC-Exos treatment repaired ocular surface damage. Additionally, mADSC-Exos inhibited cell apoptosis, decreased the levels of interleukin (IL)-1 $\beta$, IL-6, IL-1 $a$, interferon (IFN)- $\gamma$, and tumor necrosis factor (TNF)- $a$, and increased levels of the anti-inflammatory cytokine IL-10. Meanwhile, NLRP3 inflammasome activation and upregulation of caspase-1, IL-1 $\beta$, and IL-18 were reversed by mADSC-Exos. Conclusions: mADSC-Exos alleviate ocular surface inflammation, suggesting that it is a promising treatment for dry eye.

(c) 2021 The Author(s)

Published by S. Karger AG, Basel karger@karger.com www.karger.com/ore

Karger $\stackrel{\text { ' }}{5}$

BOPEN ACCESS
(C) 2021 The Author(s)

Published by S. Karger AG, Basel

This is an Open Access article licensed under the Creative Commons Attribution-NonCommercial-4.0 International License (CC BY-NC) (http://www.karger.com/Services/OpenAccessLicense), applicable to the online version of the article only. Usage and distribution for commercial purposes requires written permission.
Correspondence to:

Guifang Wang, lafangme@ldzxyy.com 


\section{Introduction}

Dry eye, one of the most common diseases in ophthalmology, is characterized by loss of tear film homeostasis and ocular surface symptoms [1]. Moderate to severe dry eye can cause irritation, fatigue, chronic pain, and other types of discomfort, which can reduce patients' work productivity and quality of life and, in extreme cases, lead to psychological problems such as anxiety and depression [2]. Although the initial cause of dry eye is different, inflammation plays a vital role in the pathogenesis of dry eye. A large number of pro-inflammatory factors such as tumor necrosis factor (TNF)- $\alpha$, interleukin (IL)- $1 \alpha$, IL$1 \beta$, IL-6, IL-8, IL-12, IL-13, interferon (IFN)- $\gamma$, and macrophage inflammatory protein (MIP)-1 $\alpha$ are present in ocular surface tissue and tears of patients with dry eye; these activate inflammation-related signal transduction pathways, thereby aggravating the inflammatory immune response on the ocular surface, which results in tear instability and increased osmotic pressure [3, 4].

Adipose-derived mesenchymal stem cells (ADSCs) have anti-inflammatory, immunomodulatory, tissue reparative, and neuroprotective effects that are exerted through paracrine release of bioactive factors in various autoimmune and inflammatory diseases $[5,6]$. ADSCs have been used in the treatment of multiple ocular diseases, including glaucoma [7], corneal chemical burns [8], reconstruction of the ocular surface [9], thyroid-associated orbitopathy [10], age-related macular degeneration [11], and optic nerve injury [12]. ADSC-derived exosomes (ADSC-Exos) containing bioactive molecules such as mRNAs, microRNAs, and functional proteins mediate the paracrine action of ADSCs [13]. Thus, ADSC-Exos have functions similar to those of ADSCs but without the associated risks of immune rejection, malignant transformation, and microvascular occlusion [14]. Exosomes are being explored as a new biomarker and therapeutic tool for dry eye [15-17].

Tear film hyperosmolarity induces chronic ocular surface inflammation; the innate immune response of the ocular surface epithelium triggers NLR family pyrin domain-containing 3 (NLRP3) inflammasome activation, leading to increased IL-1 $\beta$ secretion [18]. The expression of the NLRP3 inflammasome and downstream inflammatory factors including caspase-1, IL- $1 \beta$, and IL-18 was shown to be elevated in dry eye patients or animal models $[19,20]$. The imbalance of the NLRP3 inflammasome can exacerbate ocular surface damage, while its inhibition alleviated dry eye symptoms and ocular surface inflammation [21, 22]. Moreover, MSC-derived exosomes reduced inflammatory factor secretion by suppressing NLRP3 inflammasome activation [23, 24]. These results suggest that the NLRP3 inflammasome plays an important role in dry eye progression.

In this study, we investigated whether topical application of mouse ADSC-Exos (mADSC-Exos) had therapeutic benefits for dry eye using a mouse model. We also aimed to get insight into the molecular and signaling mechanisms of mADSC-Exos.

\section{Materials and Methods}

\section{Cell Culture}

Mouse ADSCs (mADSCs) were grown in a complete stem cell culture medium in a humidified atmosphere of $5 \% \mathrm{CO}_{2}$ and $95 \%$ air at $37^{\circ} \mathrm{C}$. After $24 \mathrm{~h}$ of culture, the supernatant was collected and processed for nanoparticle tracking analysis (NTA), transmission electron microscopy, and Western blotting.

\section{Experimental Animals}

Male C57BL/6 mice (6-8 weeks old, weighing 22-24 g) were purchased from Guangdong Medical Laboratory Animal Center (Guangzhou, China). The animals were housed 3-5 per cage with free access to water and food at $22 \pm 2^{\circ} \mathrm{C}$ and $50 \pm 10 \%$ humidity on a 12:12-h light/dark cycle. All procedures involving mice were performed in accordance with guidelines of the experimental animal Ethics Committee of Guangzhou University of Traditional Chinese Medicine. At the end of the experiment, 6 mice in each group were randomly selected, and the cornea and conjunctiva of the left eye were removed and embedded in an optimal cutting temperature medium (Tissue-Tek, Elkhart, IN, USA) and sectioned at a thickness of $6 \mu \mathrm{m}$. The sections were used for hematoxylin and eosin (HE) and periodic acid-Schiff (PAS) staining and the terminal deoxynucleotidyl transferase dUTP nick-end labeling (TUNEL) assay. The cornea and conjunctiva of the right eye were used for quantitative real-time (qRT) PCR, Western blotting, and flow cytometry.

\section{Isolation of $M A D S C$-Exos}

The supernatant of mADSC cultures was collected and centrifuged at $300-500 \mathrm{~g}$ for $10 \mathrm{~min}$ at $4^{\circ} \mathrm{C}$ to remove dead cells and cell debris. Then, the supernatant was centrifuged at $2,000 \mathrm{~g}$ for $20 \mathrm{~min}$ at $4^{\circ} \mathrm{C}$ to remove biopolymers and apoptotic bodies. The supernatant was filtered through a $0.22-\mu \mathrm{m}$ pore filter (Corning, Corning, NY, USA) and ultracentrifuged at $100,000 \mathrm{~g}$ for $1 \mathrm{~h}$ at $4^{\circ} \mathrm{C}$ (Type 70.1 Ti fixed-angle ultracentrifuge rotor; Beckman Coulter, Brea, CA, USA). The pellet was resuspended in $50 \mu \mathrm{L}$ phosphate-buffered saline (PBS; Gibco, Grand Island, NY, USA). Aliquots of $\mathrm{mADSC}$-Exos were stored at $-80^{\circ} \mathrm{C}$ in Eppendorf tubes.

Nanoparticle Tracking Analysis

The NTA was performed using a nanoparticle tracking analyzer (NanoSight NS300; Malvern Instruments, Malvern, UK).

Transmission Electron Microscopy

mADSC-Exos $(5 \mu \mathrm{L})$ were added to formvar/carbon-coated copper grids, dried for $20 \mathrm{~min}$, then briefly washed with ultrapure water, and stained with $1 \%$ uranyl acetate. The samples were visu- 
Table 1. qRT-PCR primer sequences

\begin{tabular}{lll}
\hline Gene & Sense & Antisense \\
\hline NLRP3 & 5'-TCTGCACCCGGACTGTAAAC-3' & 5'-CATTGTTGCCCAGGTTCAGC-3' \\
Caspase-1 & 5'-GAAACGCCATGGCTGACAAG-3' & 5'-GATCACATAGGTCCCGTGCC-3' \\
IL-1 & 5'-GTCGCTCAGGGTCACAAGAA-3' & 5'-GTGCTGCCTAATGTCCCCTT-3' \\
IL-18 & 5'-GGCTGCCATGTCAGAAGACT-3' & 5'-GTCTGGTCTGGGGTTCACTG-3' \\
18sRNA & 5'-GTAACCCGTTGAACCCCATT-3' & 5'-CCATCCAATCGGTAGTAGCG -3' \\
\hline
\end{tabular}

IL, interleukin; qRT-PCR, quantitative real-time PCR; NLRP3, NLR family pyrin domaincontaining 3 .

alized with a TE microscope (model 1010; JEOL, Tokyo, Japan) operated at $90 \mathrm{kV}$, and images were captured with a Soft Imaging Veleta digital camera (Olympus, Tokyo, Japan).

\section{Flow Cytometry}

mADSCs $\left(6 \times 10^{5}\right.$ cells $)$ were collected and washed in PBS containing $0.1 \%$ bovine serum albumin and $0.01 \%$ sodium azide; they were then incubated for $30 \mathrm{~min}$ at $4^{\circ} \mathrm{C}$ with FITC-conjugated anticluster of differentiation (CD) 44 (eBioscience, San Diego, CA, USA, 1:100), PE-conjugated anti-CD34 (Invitrogen, Waltham, MA, USA, 1:100), APC-conjugated anti-CD29 (eBioscience, 1:100), and PerCP-Cy5.5-conjugated anti-SCA-1 (eBioscience, 1:100) antibodies. After 3 washes in PBS and fixing with $0.1 \%$ formaldehyde, the cells were analyzed on a FACScan flow cytometer (Becton Dickinson, Franklin Lakes, NY, USA).

The cornea and conjunctiva were homogenized in lysis buffer and centrifuged at $12,000 \mathrm{~g}$ for $5 \mathrm{~min}$ at $4^{\circ} \mathrm{C}$. The supernatant was then collected. Levels of pro-inflammatory cytokine were measured using BioLegend LEGENDplex ${ }^{\mathrm{TM}}$ multiplex bead-based assay from BioLegend (San Diego, CA, USA).

\section{Experimental Dry Eye Model}

An experimental dry eye model was established in mice by topical application of benzalkonium chloride (BAC). The eyes of the mice were instilled with $5 \mu \mathrm{L}$ of $0.2 \%$ BAC twice daily (at 9:00 a.m. and 4:00 p.m.) for 14 days, except for the sham group, which was instilled with an equal volume of PBS. A total of 36 mice were randomly divided into sham; model (0.2\% BAC); $12.5,25$, and $50 \mathrm{mg}$ / $\mathrm{mL}$ mADSC-Exos; and positive control ( $0.1 \%$ pranoprofen) groups (6 mice each). At 14 days after BAC treatment, the eyes of mice were instilled with $5 \mu \mathrm{L}$ mADSC-Exos or $0.1 \%$ pranoprofen 3 times a day for 7 days, while the sham and model groups were instilled with an equal volume of PBS.

\section{Fluorescein Staining}

Fluorescein staining was performed by applying 1\% fluorescein solution to the conjunctiva on days 4 and 7 after mADSCExos treatment. The corneal surface was examined with a slit-lamp microscope (Haag-Streit Diagnostics, Wedel, Germany) and divided into 4 areas that were separately scored in a blinded fashion on a scale from 0 to 4 according to the 2017 Tear Film and Ocular Surface Society Dry Eye Workshop II grading system [25].

\section{Measurement of Tear Volume}

After anesthetization with isoflurane, tear volume was measured with phenol red-impregnated cotton threads (Zone-Quick; Yokota, Tokyo, Japan). The lower eyelid was pulled down slightly, and a 1-mm length of the thread was placed on the palpebral conjunctiva for $15 \mathrm{~s}$. The length of the wet red thread was measured in millimeters. The test was repeated 3 times for each eye, and the average value was taken as the final result.

\section{Measurement of Tear Film Break-Up Time (BUT)}

After anesthetization with isoflurane, tear film BUT on the ocular surface was measured. Fluorescein sodium (1\%) was instilled in the conjunctival sac, and the eyelids were manually closed 3 times. The ocular surface was examined under a slit-lamp microscope. At the first appearance of dryness, BUT was recorded (in seconds). The test was repeated 3 times for each eye, and the average value was taken as the final result.

\section{Hematoxylin-Eosin (HE) and Periodic Acid-Schiff (PAS)}

Staining

Frozen tissue sections were stained using the HE and PAS staining kits according to the manufacturer's protocols. Mucinfilled goblet cells were counted in 6 representative sections at corresponding positions in each sample.

\section{Terminal Deoxynucleotidyl Transferase dUTP Nick End}

Labeling (TUNEL) Assay

The TUNEL assay kit was used according to the manufacturer's instructions to detect apoptotic cells in the cornea. Cell nuclei were counterstained with DAPI for $10 \mathrm{~min}$, and the cells were visualized with a laser scanning confocal microscope (TCS SP5; Leica, Wetzlar, Germany). TUNEL-positive cells were calculated from 3 random fields at $\times 100$ magnification for each treatment group.

\section{Western Blotting}

mADSC-Exos, cornea, and conjunctiva were separately homogenized in radioimmunoprecipitation lysis buffer containing 1 $\mu \mathrm{g} / \mathrm{mL}$ phenylmethylsulfonyl fluoride and $1 \mu \mathrm{L}$ phosphatase inhibitor cocktail on ice. The precipitate was removed by centrifugation at $12,000 \mathrm{~g}$ for $30 \mathrm{~min}$ at $4^{\circ} \mathrm{C}$. The total protein concentration of the supernatant was determined with the BCA protein assay kit. Samples containing $30 \mu \mathrm{g}$ of protein were separated by $10-15 \%$ $(\mathrm{w} / \mathrm{v})$ sodium dodecyl sulfate-polyacrylamide gel electrophoresis and transferred to a polyvinylidene difluoride membrane that was blocked in Tris-buffered saline with $0.1 \%$ Tween-20 and 5\% (v/v) bovine serum albumin at room temperature for $2 \mathrm{~h}$ and then incubated overnight at $4^{\circ} \mathrm{C}$ with antibodies against CD9, CD63, CD81, NLRP3, caspase-3, PI3K, p-PI3K, p65, p-p65, and loading control GAPDH (Cell Signaling Technology, Danvers, MA, USA), all used at 1:1,000 dilution. The following day, the membrane was incubated with HRP-conjugated secondary antibody (Cell Signaling
42 
Fig. 1. Representative photomicrographs of flow cytometry for the mADSC-positive markers (CD44, CD34, Sca-1, and CD29). mADSC, mouse adipose-derived mesenchymal stem cell; CD, cluster of differentiation.

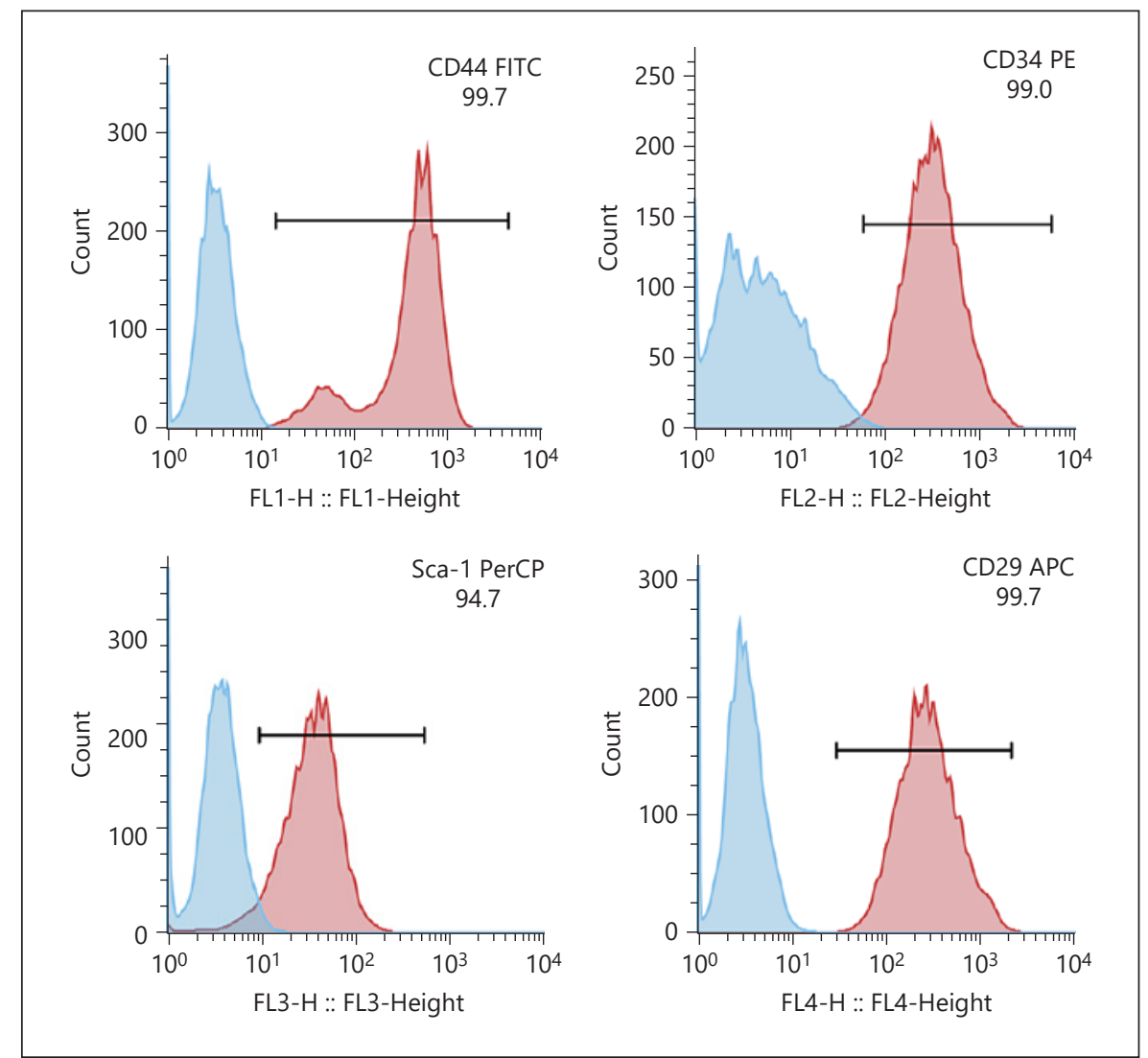

Technology, 1:2,000) in Tris-buffered saline with $0.1 \%$ Tween-20 for $2 \mathrm{~h}$ at room temperature. Protein bands were visualized with the ECL Advance ${ }^{\mathrm{TM}}$ Western blotting detection kit, and densitometric analysis was performed using Care Stream Molecular Imaging software (Carestream Health, New Haven, CT, USA).

Quantitative Real-Time PCR ( $q R T-P C R$ )

The total RNA was extracted from the cornea and conjunctiva with TRIzol reagent (Thermo Fisher Scientific, Waltham, MA, USA). Total RNA ( $1 \mu \mathrm{g})$ was reversely transcribed using M-MuLv Reverse Transcriptase (New England Biolabs, Beverly, MA, USA). Quantitative PCRs were performed in triplicates for all samples using the Mx 3005P Real-Time PCR system (Stratagene) with SYBR Green Supermix (Thermo Fisher Scientific). The reaction conditions were as follows: $98^{\circ} \mathrm{C}$ for $30 \mathrm{~s}$, followed by 40 cycles of $98^{\circ} \mathrm{C}$ for $10 \mathrm{~s}, 60^{\circ} \mathrm{C}$ for $30 \mathrm{~s}$, and $72^{\circ} \mathrm{C}$ for $15 \mathrm{~s}$ in a $20 \mu \mathrm{L}$ reaction volume. $\Delta \mathrm{Ct}$ values for target genes were calculated relative to the levels of $18 \mathrm{~S}$ rRNA transcript. All primers for qRT-PCR were validated before use based on the melting curve and $C_{\mathrm{t}}$ value. Primers used for qRT-PCR analysis are listed in Table 1.

\section{Statistical Analysis}

Data were analyzed with Prism v8.0 software (GraphPad, La Jolla, CA, USA). Results are expressed as mean \pm SEM. Differences between groups were assessed by one-way analysis of variance followed by Dunnett's multiple comparisons tests; differences with $p<0.05$ were considered significant.

mADSC-Exos Inhibit NLRP3 Activation in Dry Eye

\section{Results}

Characterization of $m A D S C$ s and $m A D S C$-Exos

mADSCs were positive for CD29, CD34, CD44, and SCA-1 expression (>70\%) (Fig. 1). mADSC-Exos were extracted by ultracentrifugation and characterized by Western blotting, NTA, and transmission electron microscopy. The mADSC-Exos expressed CD9, CD63, and CD81, with an average particle size of $134 \mathrm{~nm}$ (range: $40-$ $600 \mathrm{~nm}$ ). The mADSC-Exos had a teacup-like morphology with a bilayer membrane (Fig. 2).

\section{mADSC-Exos Improve Tear Film Stability and}

\section{Alleviate Corneal Surface Damage}

To investigate the effects of mADSC-Exos on dry eye, we performed fluorescein staining of the ocular surface and evaluated tear film BUT (Fig. 3a-g). The fluorescein staining score was significantly higher in the model group than in the sham group on days 4 and 7 after mADSCExos treatment. However, the mADSC-Exos group (50 $\mathrm{mg} / \mathrm{mL}$ ) had a lower score than the model group, indicating that there was less BAC-induced corneal damage. Similarly, tear volume and tear film BUT were reduced in 


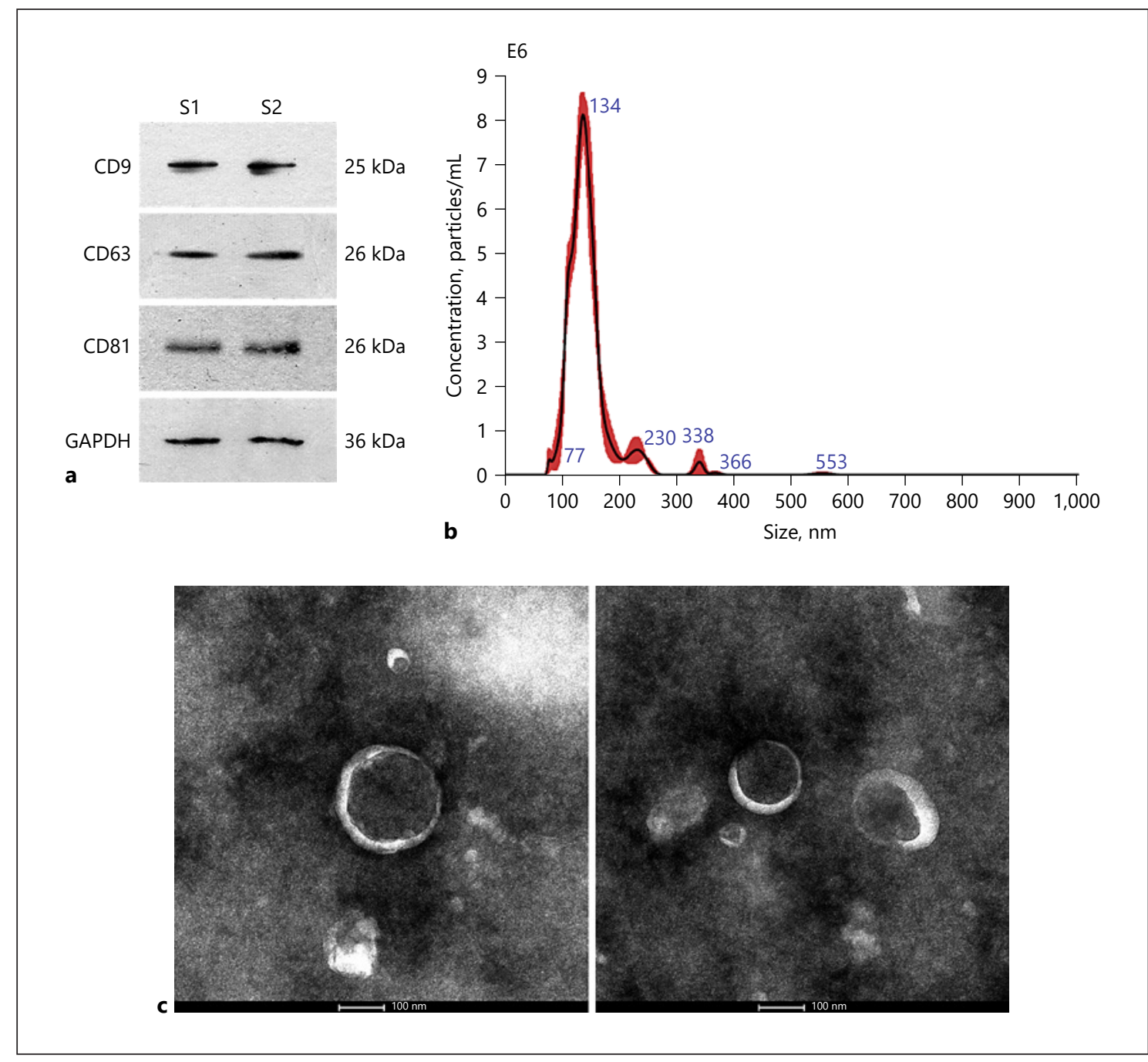

Fig. 2. mADSC-Exos determination. a Western blot of CD9, CD63, and CD81 expression in mADSC-Exos. b NTA of mADSC-Exos size. c Representative photomicrographs of TEM for the mADSC-Exos. Scale bar, $100 \mathrm{~nm}$. NTA, nanoparticle tracking analysis; TEM, transmission electron microscopy; mADSC-Exos, mouse adiposederived mesenchymal stem cell-derived exosomes; CD, cluster of differentiation.

the model group compared to the sham group (Fig. 3dg), but treatment with mADSC-Exos increased these values relative to the model group: in the $50 \mathrm{mg} / \mathrm{mL} \mathrm{mAD}$ $\mathrm{SC}$-Exos group, they were comparable to the positive control.

Histologic analysis of the conjunctival tissue was performed by HE staining (Fig. 4). Compared to the sham group, conjunctival epithelial cells in the model group had irregular morphology; however, this was alleviated in the positive and $50 \mathrm{mg} / \mathrm{mL}$ mADSCs-Exos-treated groups. Thus, mADSCs-Exos protect against conjunctival damage associated with dry eye.
mADSC-Exos Exert a Protective Effect against Dry Eye by Preserving Goblet Cells in the Conjunctiva

The effect of mADSC-Exos on the conjunctival epithelium was evaluated by PAS staining and quantification of positively stained mucin-filled goblet cells in the conjunctiva (Fig. 5). The number of PAS-stained cells was decreased in the BAC-induced dry eye condition, but the trend was reversed in the $50 \mathrm{mg} / \mathrm{mL} \mathrm{mADSC}$-Exos group (Fig. 5). We also detected apoptotic cells with the TUNEL assay and found that the model group had an increased number of TUNEL-positive cells compared to the control group (Fig. 6a, b), while mADSC-Exos treatment (50 mg/ 


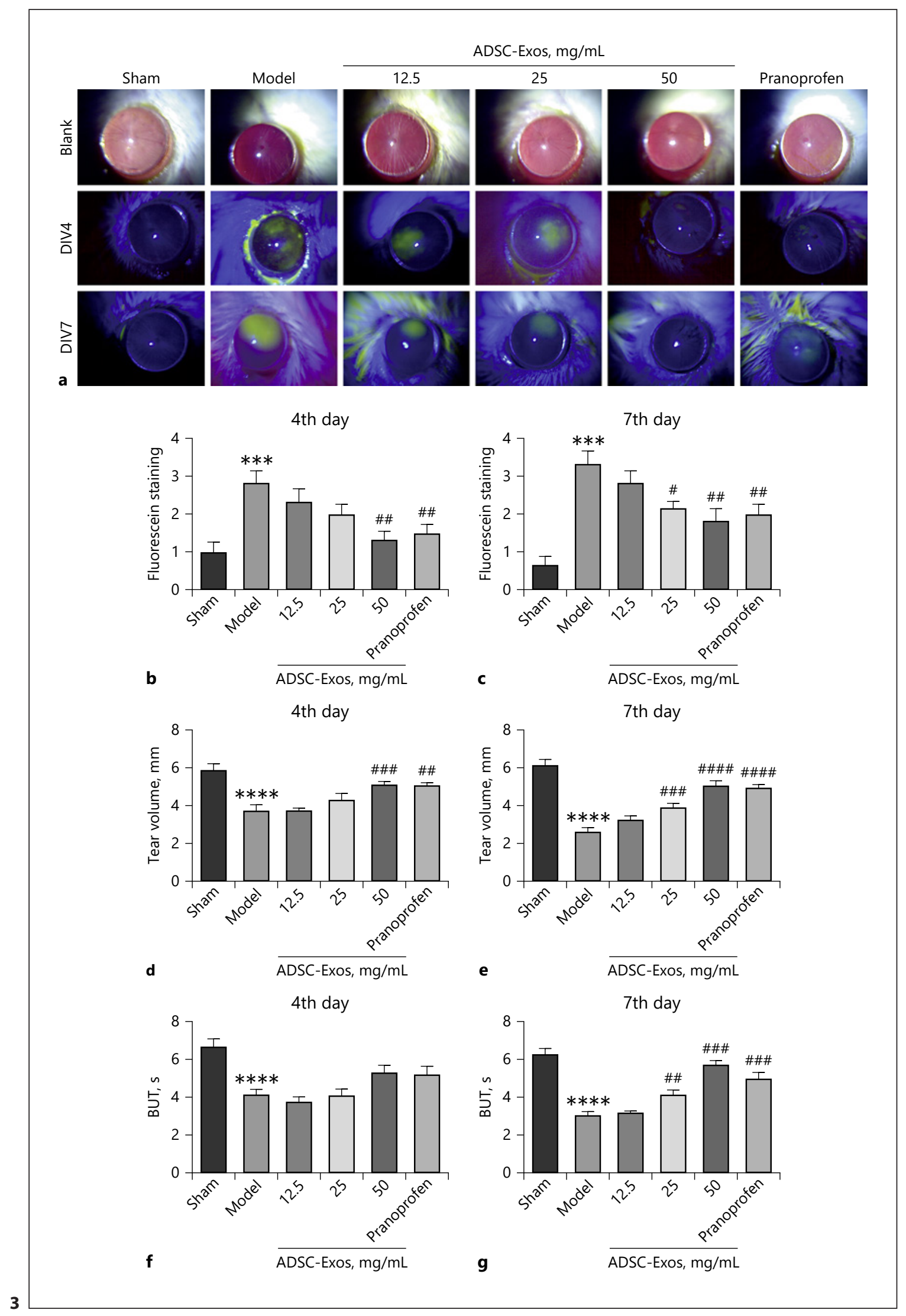

(For legend see next page.) 


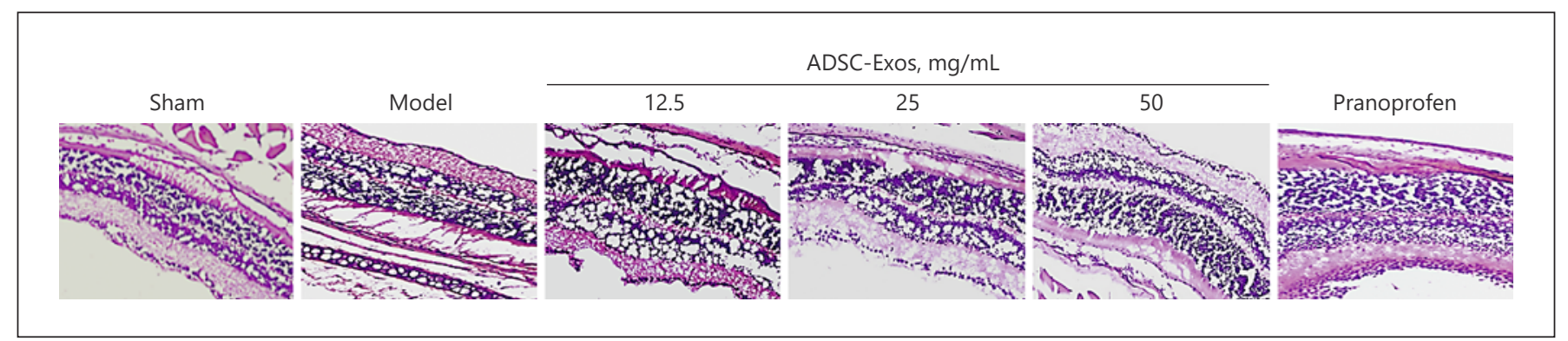

Fig. 4. Representative images of conjunctival epithelium integrity (magnification, $\times 100$ ). ADSC-Exos, ADSC-derived exosomes.

Fig. 5. $\mathrm{mADSC}$-Exos promoted the recovery of BAC-induced conjunctival injury. a Representative PAS staining of conjunctival goblet cells. Scale bar, $100 \mu \mathrm{m}$. b Comparison of goblet cell density. Data are shown as mean \pm SEM of 3 mice per group. ${ }^{* * * *} p<0.0001$ versus sham group. ${ }^{\# \#} p<$ $0.01,{ }^{\# \#} p<0.001$ versus the model group. PAS, periodic acid-Schiff; mADSC-Exos, mouse adipose-derived mesenchymal stem cell-derived exosomes; BAC, benzalkonium chloride; ADSC-Exos, ADSC-derived exosomes.

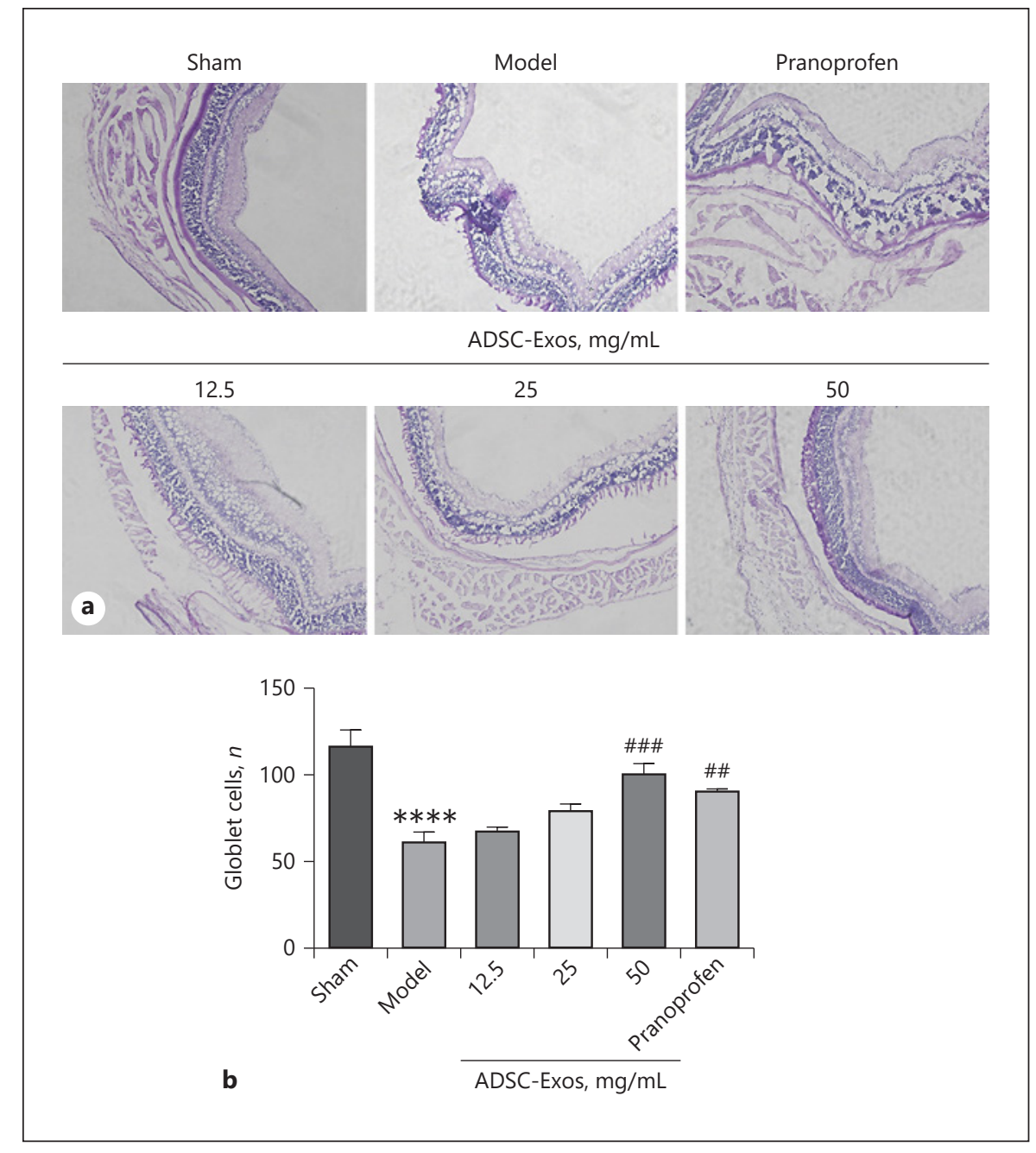

Fig. 3. mADSC-Exos protect the ocular surface in BAC-induced murine dry eye model. a Corneal fluorescein staining. b, c Fluorescein staining. d, e Tear volume. f, $\mathbf{g}$ Tear film BUT. Data are presented as mean \pm SEM, $n=6 .{ }^{* * *} p<0.001$ and ${ }^{* * * *} p<0.0001$ versus sham group. ${ }^{\#} p<0.05,{ }^{\# \#} p<0.01,{ }^{\# \#} p<0.001$, and ${ }^{\# \# \#} p<$
0.0001 versus model group. mADSC-Exos, mouse adipose-derived mesenchymal stem cell-derived exosomes; BAC, benzalkonium chloride; BUT, breakup time; ADSC-Exos, ADSC-derived exosomes. 


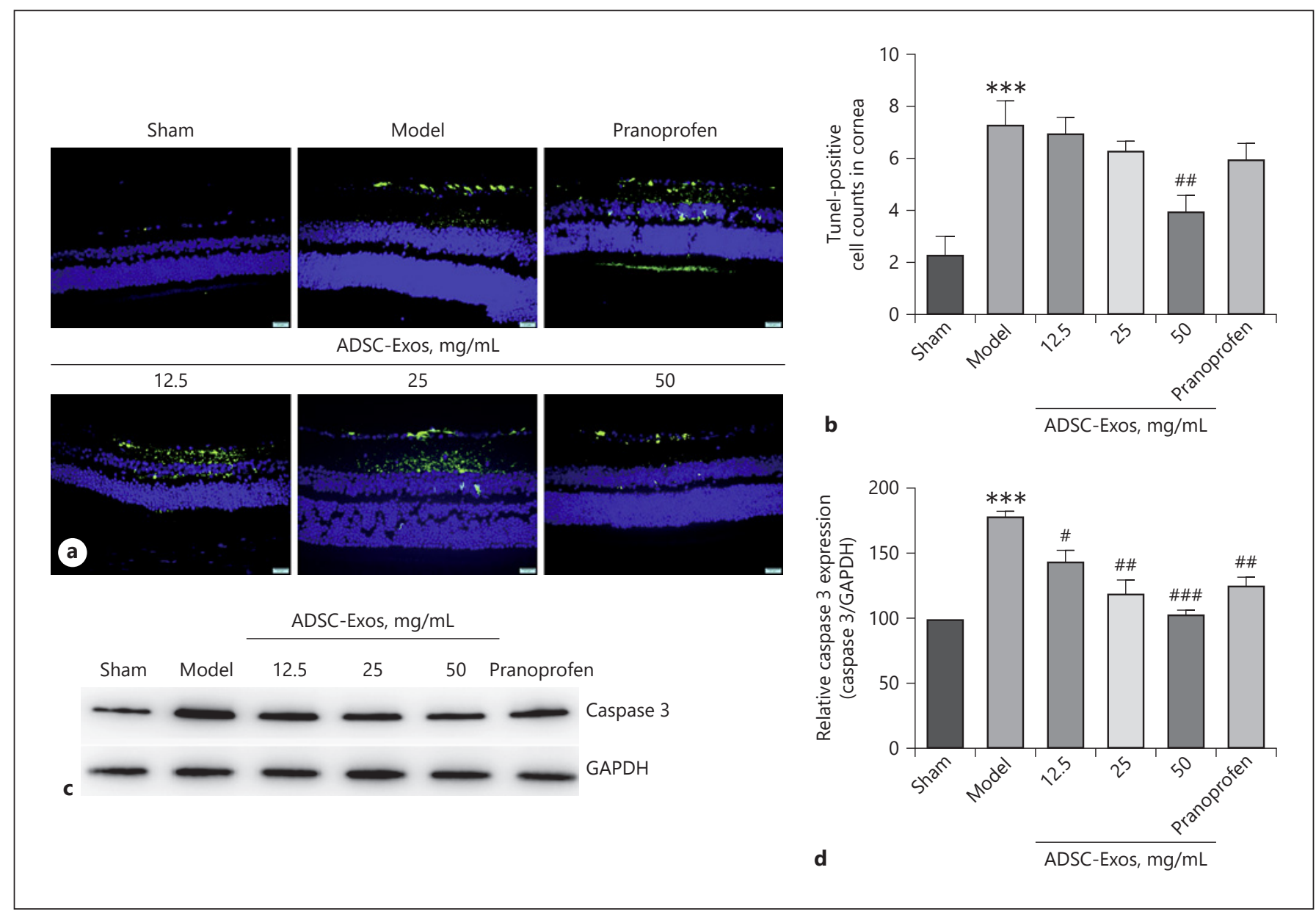

Fig. 6. mADSC-Exos reduce apoptosis of BAC-induced conjunctival cells. a Representative photomicrographs of TUNEL staining (green color) for apoptosis cells with DAPI staining (blue color) for nuclei. Scale bars, $20 \mu \mathrm{m}$. b Numbers of TUNEL-positive cells. c Western blot for caspase-3 expression. d Intensity of caspase-3 in the conjunctiva. Data are shown as mean \pm SEM of 3 mice per

$\mathrm{mL}$ ) reduced the number. These data demonstrate that mADSC-Exos treatment preserves the conjunctiva in a mouse dry eye model.

\section{mADSC-Exos Suppress BAC-Induced Inflammation}

We next evaluated the effects of mADSCs-Exos on inflammatory cytokine production in the cornea and conjunctiva. BAC installation caused an increase in the levels of pro-inflammatory cytokines including IL- $1 \beta$, IL-6, IL$1 \alpha$, IFN- $\gamma$, and TNF- $\alpha$ in both tissues; however, mADSCExos reduced the levels while increasing that of the antiinflammatory cytokine IL-10 (Fig. 7a-f), similar to the effect observed with the positive control ( $0.1 \%$ pranoprofen).

mADSC-Exos Inhibit NLRP3 Activation in Dry Eye group. ${ }^{* * *} p<0.001$ versus sham group. ${ }^{\#} p<0.05,{ }^{\# \#} p<0.01,{ }^{\# \# \#} p<$ 0.001 versus model group. TUNEL, terminal deoxynucleotidyl transferase dUTP nick-end labeling; mADSC-Exos, mouse adipose-derived mesenchymal stem cell-derived exosomes; BAC, benzalkonium chloride; ADSC-Exos, ADSC-derived exosomes.

\section{mADSC-Exos Suppress NLRP3 Inflammasome Activation}

Nuclear factor (NF)- $\kappa B$ signaling is induced via activation of Toll-like receptor 4 or TNF receptor, resulting in the upregulation of NLRP3, pro-IL-1 $\beta$, and pro-IL-18. $\mathrm{PI} 3 \mathrm{~K} /$ protein kinase $\mathrm{B}$ signaling acts upstream of NF- $\mathrm{B}$ [26]. Here, we examined the effect of mADSC-Exos on NLRP3 inflammasome activation in BAC-induced dry eye by Western blotting and found that the protein levels of p-PI3K, p-p65, and NLRP3 were increased in the model group; this was reversed by $50 \mathrm{mg} / \mathrm{mL}$ mADSC-Exos treatment (Fig. 8a-d). Similar trends were observed in the mRNA levels of NLRP3, caspase-1, IL-1 $\beta$, and IL-18, as determined by qRT-PCR analysis (Fig. 8e-h). 


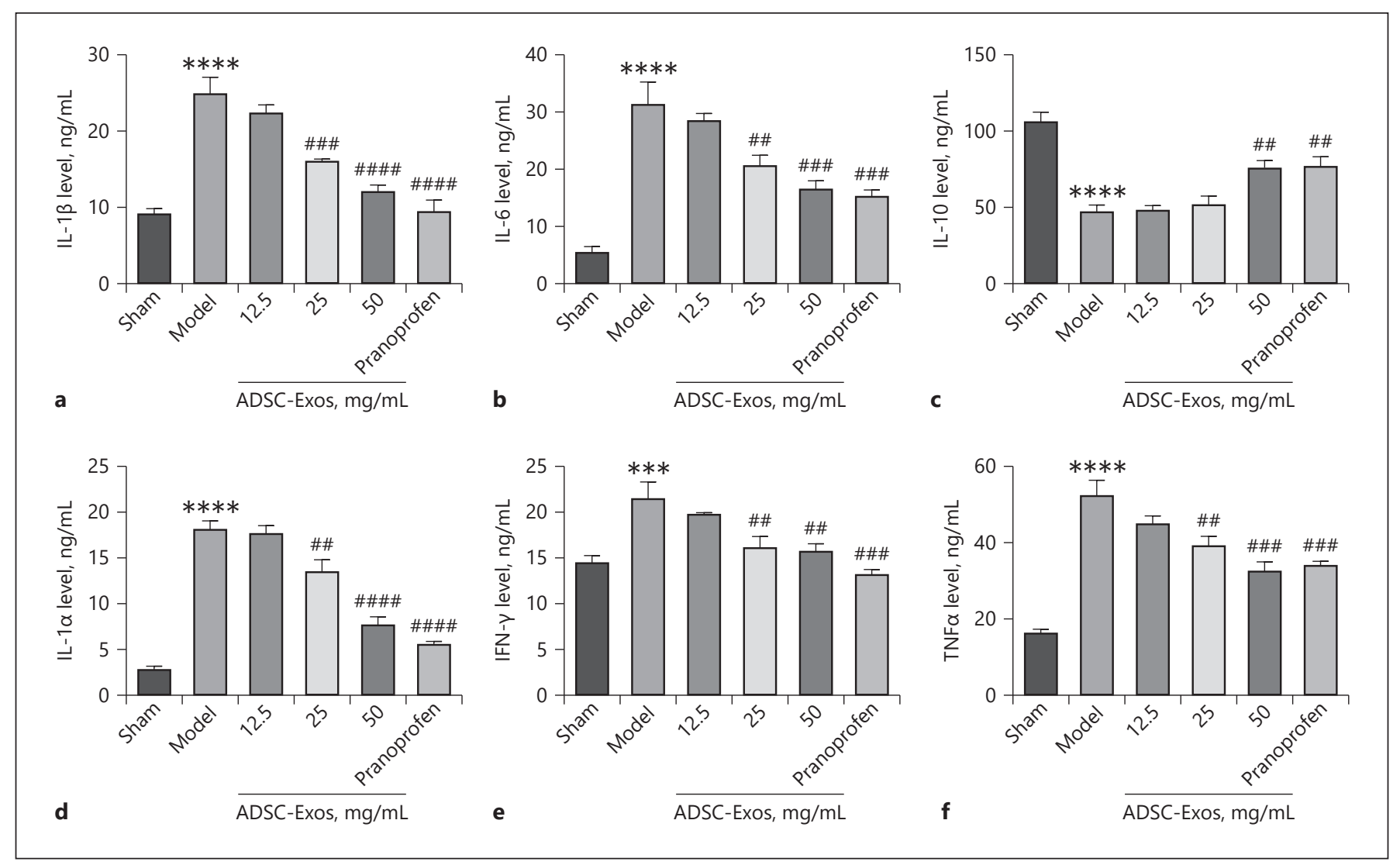

Fig. 7. mADSC-Exos alleviate BAC-induced conjunctival inflammation. Serum levels of pro-inflammatory cytokines, including IL$1 \beta$ (a), IL-6 (b), IL-10 (c), IL-1 $\alpha$ (d), IFN- $\gamma$ (e), and TNF- $\alpha$, were determined using BioLegend LEGENDplex ${ }^{\mathrm{TM}}$ pro-inflammatory chemokine and cytokine kits (f). Data are shown as mean \pm SEM of 3 mice per group. ${ }^{* * *} p<0.001$ and ${ }^{* * * *} p<0.0001$ versus sham

\section{Discussion}

Dry eye is a multifactorial disease with a high prevalence worldwide. The main treatment approaches for dry eye are artificial tears and anti-inflammatory agents; these alleviate symptoms in some patients, but the effects are relatively weak and short-lived. As such, there is a need for new therapeutic strategies for dry eye. Similar to ADSCs, ADSC-Exos can suppress inflammation, prevent apoptosis, repair injured tissue, and modulate the immune response. With their nanoscale dimensions, exosomes can readily enter target organs through various biological barriers. Rui et al. [27] demonstrated that murine olfactory ecto-mesenchymal stem cell-derived exosomes could ameliorate the development of Sjögren's syndrome by enhancing the immunosuppressive function of myeloid-derived suppressor cells. Li et al.'s [28] study also showed

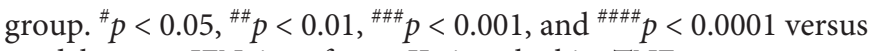
model group. IFN, interferon; IL, interleukin; TNF, tumor necrosis factor; mADSC-Exos, mouse adipose-derived mesenchymal stem cell-derived exosomes; BAC, benzalkonium chloride; ADSCExos, ADSC-derived exosomes.

that MSC-Exos had an obvious therapeutic effect on the dry eye mice model. In this study, we found that topical application of mADSC-Exos significantly improved dry eye symptoms in mice by repairing corneal epithelium, promoting tear secretion, and preserving the number of conjunctival goblet cells. Thus, topical application of mADSC-Exos has therapeutic potential for the treatment of dry eye.

The balance between tear production and discharge is very important for maintaining the steady state of the tear film; changes in tear film structure and composition lead to altered permeability and stability, resulting in ocular surface inflammation and corneal sensory nerve abnormalities, which promote the development of dry eye [29]. In our study, topical application of mADSC-Exos (50 mg/ $\mathrm{mL}$ ) to the ocular surface enhanced the integrity of tear film and stimulated tear secretion while also preserving 


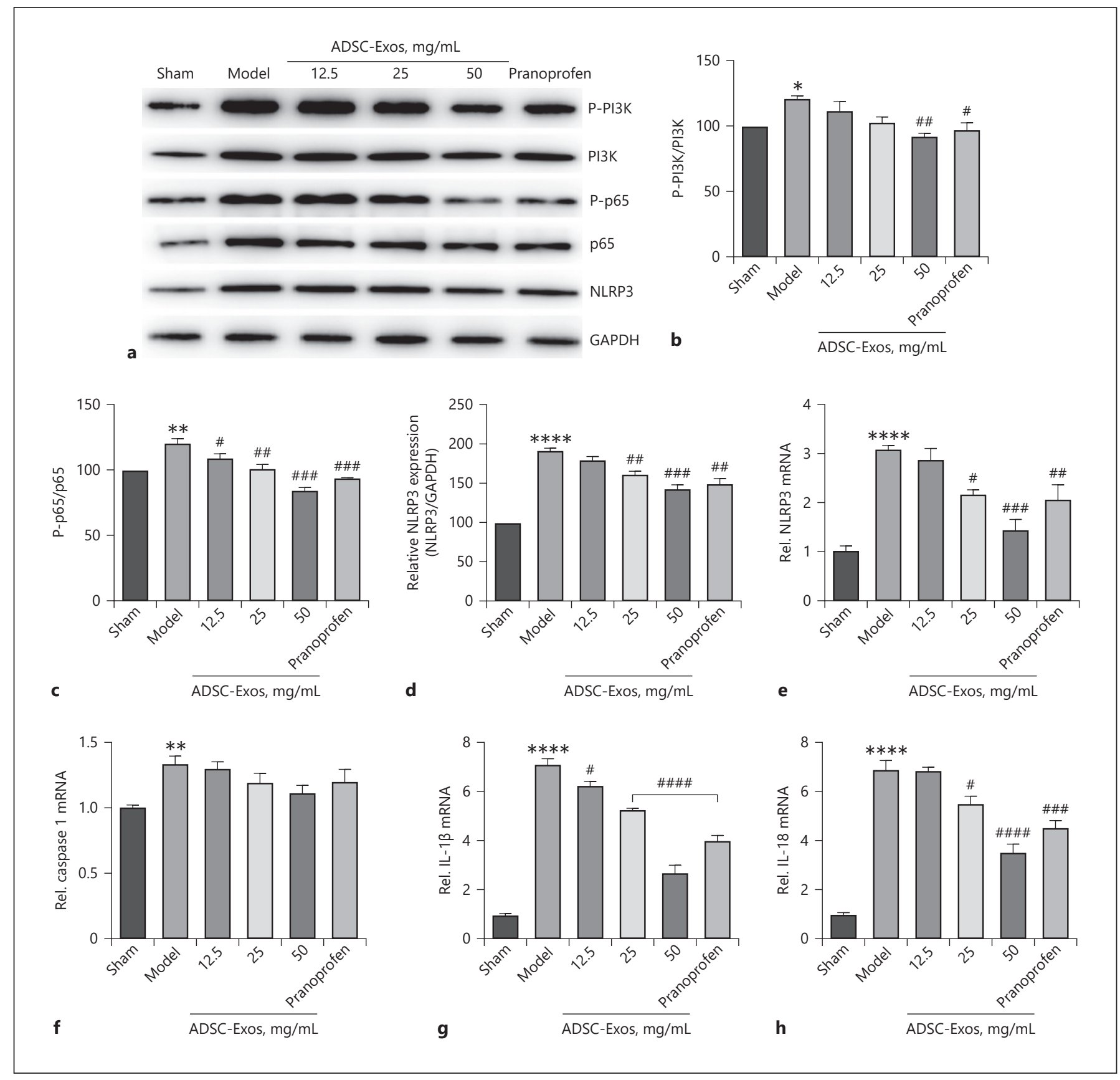

Fig. 8. mADSC-Exos prevent NLRP3 inflammasome activation in BAC-induced mouse dry eye model. Western blotting (a) and quantitative analysis (b-d) of p-PI3K/PI3K, p-p65/p65, and NLRP3 in cornea and conjunctiva. RNA from cornea and conjunctiva of each mouse were purified and converted into cDNA. mRNA expression levels of NLRP3 (e), caspase-1 (f), IL-1 $\beta$ (g), and IL-18 (h) in cornea and conjunctiva were determined using qRT-PCR. Data are shown as mean \pm SEM of 3 mice per group. ${ }^{*} p<0.05,{ }^{* *} p$
$<0.01$, and ${ }^{* * * *} p<0.0001$ versus sham group. ${ }^{\#} p<0.05,{ }^{\# \#} p<0.01$, $\# \#$ \#\# 0.001 , and ${ }^{\# \# \# ~} p<0.0001$ versus model group. IFN, interferon; IL, interleukin; mADSC-Exos, mouse adipose-derived mesenchymal stem cell-derived exosomes; BAC, benzalkonium chloride; NLRP3, NLR family pyrin domain-containing 3; qRT-PCR, quantitative real-time PCR; ADSC-Exos, ADSC-derived exosomes. 
the number of mucin-filled goblet cells in the conjunctiva and inhibiting the apoptosis of corneal epithelial cells in mice with BAC-induced dry eye. Moreover, mADSCExos treatment reduced IL- $1 \beta$ and IL- 6 levels in the cornea and conjunctiva in the dry eye model. IL- $1 \beta$ and IL- 6 are pro-inflammatory cytokines secreted by ocular surface epithelial cells in response to hyperosmotic stress; their downregulation was shown to mitigate ocular surface epithelium injury and prevent epithelial cell loss [17, $30,31]$.

The NLRP3 inflammasome plays an important role in innate immunity. Hyperosmolar stress increases NLRP3 inflammasome activation in conjunctival tissue, which promotes the release of IL- $1 \beta$ and IL-18 and contributes to inflammation $[18,19]$. The activation of the NLRP3/ IL- $1 \beta$ signaling axis induced by hypertonic stress is a key step in epithelial inflammation and dry eye progression $[32,33]$. It was previously reported that MSC-derived exosomes prevented acute liver failure by suppressing NLRP3 inflammasome activation in macrophages [23]. In the current study, we found that mRNA and protein levels of NLRP3 inflammasome components were upregulated by BAC-induced dry eye, with a corresponding increase in the expression of the downstream inflammatory factors caspase-1, IL-1 $\beta$, and IL-18. However, treatment with mADSC-Exos significantly reduced the mRNA and protein expression of NLRP3, caspase- 1 , IL- $1 \beta$, and IL-18 in the conjunctiva. This is consistent with the finding that extracellular vesicles derived from adipose tissue stem cells suppressed the NLRP3-associated inflammatory response and promoted ocular surface repair in a desiccating stress-induced mouse dry eye model [34]. These findings along with our results demonstrate that mADSCExos suppress inflammation, promote ocular tissue repair in dry eye, and are thus a promising treatment for this disease.

\section{Conclusions}

In conclusion, our study provided convincing evidence that mADSC-Exos promoted ocular surface epithelial repair, restored goblet cells, increased tear secretion, and prevented the production of inflammatory cytokines by inhibiting the NLRP3-IL-1 $\beta$ signaling pathway. Our data strongly suggested that mADSC-Exos could be used as a potential treatment of dry eye.

\section{Acknowledgments}

We thank Prof. Nie Binghua for his critical reading and editing of the manuscript. Many thanks also to Baojian Guo for editing the manuscript.

\section{Statement of Ethics}

All procedures involving mice were performed in accordance with guidelines of the experimental animal Ethics Committee of Guangzhou University of Traditional Chinese Medicine (approval No. [2021812]).

\section{Conflict of Interest Statement}

The authors have no conflicts of interest to declare.

\section{Funding Sources}

This study was supported by the Natural Science Foundation of Hunan Province (grant No. 2020JJ5460), Technical Innovation Guidance Plan of Hunan Province (grant No. 2017SK51206), and Project of Loudi Science and Technology Bureau (grant No. [2019] No. 61).

\section{Author Contributions}

Guifang Wang designed and performed the experiments and analyzed the data; Honghui Li directed the project and analyzed the data; Hongmei Long designed the experiments and wrote the manuscript; Xileyuan Gong and Shufang Hu performed the mice experiments; and Can Gong performed the qRT-PCR and immunohistochemistry staining assays. All authors have read and approved the final manuscript.

\section{Data Availability Statement}

All data generated or analyzed during this study are included in this article. Further inquiries can be directed to the corresponding author.

\footnotetext{
References 1 Craig JP, Nichols KK, Akpek EK, Caffery B, Dua HS, Joo CK, et al. TFOS DEWS II definition and classification report. Ocul Surf. 2017 Jul;15(3):276-83.

2 Na KS, Han K, Park YG, Na C, Joo CK. Depression, stress, quality of life, and dry eye disease in Korean women: a Population-Based Study. Cornea. 2015 Jul;34(7):733-8.

3 Zhao H, Li Q, Ye M, Yu J. Tear luminex analysis in dry eye patients. Med Sci Monit. 2018 Oct;24:7595-602.
}

Wang/Li/Long/Gong/Hu/Gong 
4 Gao Y, Min K, Zhang Y, Su J, Greenwood M, Gronert K. Female-specific downregulation of tissue polymorphonuclear neutrophils drives impaired regulatory $\mathrm{T}$ cell and amplified effector $\mathrm{T}$ cell responses in autoimmune dry eye disease. J Immunol. 2015 Oct;195(7): 3086-99.

5 Shi Y, Wang Y, Li Q, Liu K, Hou J, Shao C, et al. Immunoregulatory mechanisms of mesenchymal stem and stromal cells in inflammatory diseases. Nat Rev Nephrol. 2018 Aug; 14(8):493-507.

6 Riazifar M, Mohammadi MR, Pone EJ, Yeri A, Lässer C, Segaliny AI, et al. Stem cell-derived exosomes as nanotherapeutics for autoimmune and neurodegenerative disorders. ACS Nano. 2019 Jun;13(6):6670-88.

7 Bai L, Shao H, Wang H, Zhang Z, Su C, Dong $\mathrm{L}$, et al. Effects of mesenchymal stem cell-derived exosomes on experimental autoimmune uveitis. Sci Rep. 2017 Jun;7(1):4323.

8 Rui K, Hong Y, Zhu Q, Shi X, Xiao F, Fu H, et al. Olfactory ecto-mesenchymal stem cell-derived exosomes ameliorate murine Sjögren's syndrome by modulating the function of myeloid-derived suppressor cells. Cell Mol Immunol. $2021 \mathrm{Feb}$;18(2):440-51.

9 Mead B, Tomarev S. Bone marrow-derived mesenchymal stem cells-derived exosomes promote survival of retinal ganglion cells through miRNA-dependent mechanisms. Stem Cells Transl Med. 2017 Apr;6(4):127385.

10 Moisseiev E, Anderson JD, Oltjen S, Goswami M, Zawadzki RJ, Nolta JA, et al. Protective effect of intravitreal administration of exosomes derived from mesenchymal stem cells on retinal ischemia. Curr Eye Res. 2017 Oct; 42(10):1358-67.

11 Limoli PG, Vingolo EM, Morales MU, Nebbioso M, Limoli C. Preliminary study on electrophysiological changes after cellular autograft in age-related macular degeneration. Medicine. 2014 Dec;93(29):e355.

12 Wang S, Hou Y, Li X, Song Z, Sun B, Li X, et al. Comparison of exosomes derived from induced pluripotent stem cells and mesenchymal stem cells as therapeutic nanoparticles for treatment of corneal epithelial defects. Aging. 2020 Oct;12(19):19546-62.

13 Yu B, Zhang X, Li X. Exosomes derived from mesenchymal stem cells. Int J Mol Sci. 2014 Mar;15(3):4142-57.
14 Phinney DG, Pittenger MF. Concise review: MSC-derived exosomes for cell-free therapy. Stem Cells. 2017 Apr;35(4):851-8.

15 Cortes-Troncoso J, Jang SI, Perez P, Hidalgo J, Ikeuchi T, Greenwell-Wild T, et al. T cell exosome-derived miR-142-3p impairs glandular cell function in Sjögren's syndrome. JCI Insight. 2020 May;5(9):e133497.

16 Li N, Zhao L, Wei Y, Ea VL, Nian H, Wei R. Recent advances of exosomes in immune-mediated eye diseases. Stem Cell Res Ther. 2019 Aug;10(1):278

17 Huang Y, Li R, Ye S, Lin S, Yin G, Xie Q. Recent advances in the use of exosomes in Sjögren's syndrome. Front Immunol. 2020 Aug;11:1509.

18 Zheng Q, Ren Y, Reinach PS, Xiao B, Lu H, Zhu Y, et al. Reactive oxygen species activated NLRP3 inflammasomes initiate inflammation in hyperosmolarity stressed human corneal epithelial cells and environment-induced dry eye patients. Exp Eye Res. 2015 May;134: $133-40$.

19 Niu L, Zhang S, Wu J, Chen L, Wang Y. Upregulation of NLRP3 inflammasome in the tears and ocular surface of dry eye patients. PLoS One. 2015 May; 10(5):e0126277.

20 Chi W, Hua X, Chen X, Bian F, Yuan X, Zhang $\mathrm{L}$, et al. Mitochondrial DNA oxidation induces imbalanced activity of NLRP3/NLRP6 inflammasomes by activation of caspase- 8 and BRCC36 in dry eye. J Autoimmun. 2017 Jun; 80:65-76.

21 Chen H, Li Y, Gu J, Yin L, Bian F, Su L, et al. TLR4-MyD88 pathway promotes the imbalanced activation of NLRP3/NLRP6 via caspase-8 stimulation after alkali burn injury. Exp Eye Res. 2018 Nov; 176:59-68.

22 Park B, Jo K, Lee TG, Hyun SW, Kim JS, Kim CS. Polydatin inhibits NLRP3 inflammasome in dry eye disease by attenuating oxidative stress and inhibiting the NF- $\kappa \mathrm{B}$ pathway. Nutrients. 2019 Nov;11(11):2792.

23 Liu Y, Lou G, Li A, Zhang T, Qi J, Ye D, et al. AMSC-derived exosomes alleviate lipopolysaccharide/d-galactosamine-induced acute liver failure by miR-17-mediated reduction of TXNIP/NLRP3 inflammasome activation in macrophages. EBioMedicine. 2018 Oct;36: $140-50$.
24 Xia C, Zeng Z, Fang B, Tao M, Gu C, Zheng $\mathrm{L}$, et al. Mesenchymal stem cell-derived exosomes ameliorate intervertebral disc degeneration via anti-oxidant and anti-inflammatory effects. Free Radic Biol Med. 2019 Nov; 143:1-15.

25 Wolffsohn JS, Arita R, Chalmers R, Djalilian A, Dogru M, Dumbleton K, et al. TFOS DEWS II diagnostic methodology report. Ocul Surf. 2017 Jul;15(3):539-74.

26 Yang FM, Fan D, Yang XQ, Zhu FH, Shao MJ, $\mathrm{Li}$ Q, et al. The artemisinin analog SM934 alleviates dry eye disease in rodent models by regulating TLR4/NF- $\kappa \mathrm{B} / \mathrm{NLRP3}$ signaling. Acta Pharmacol Sin. 2021 Apr;42(4):593603.

27 Rui K, Hong Y, Zhu Q, Shi X, Xiao F, Fu H, et al. Olfactory ecto-mesenchymal stem cell-derived exosomes ameliorate murine Sjögren's syndrome by modulating the function of myeloid-derived suppressor cells. Cell Mol Immunol. 2021 Feb;18(2):440-51.

28 Li J, Zhou Y, Tan G, Wang T, Xian PP, Long Q. Therapeutic effects of mesenchymal stem cells derived exosomes on dry eye mice. Rec Adv Ophthalmol. 2019 Oct;39(10):901-5

29 Galor A, Moein HR, Lee C, Rodriguez A, Felix ER, Sarantopoulos KD, et al. Neuropathic pain and dry eye. Ocul Surf. 2018 Jan;16(1): 31-44.

30 Qu M, Qi X, Wang Q, Wan L, Li J, Li W, et al. Therapeutic effects of STAT3 inhibition on experimental murine dry eye. Invest Ophthalmol Vis Sci. 2019 Sep;60(12):3776-85.

31 Harrell CR, Markovic BS, Fellabaum C, Arsenijevic N, Djonov V, Volarevic V. The role of interleukin 1 receptor antagonist in mesenchymal stem cell-based tissue repair and regeneration. Biofactors. 2020 Mar;46(2):26375.

32 Zheng Q, Ren Y, Reinach PS, She Y, Xiao B, Hua S, et al. Reactive oxygen species activated NLRP3 inflammasomes prime environmentinduced murine dry eye. Exp Eye Res. 2014 Aug; $125: 1-8$.

33 Dai Y, Zhang J, Xiang J, Li Y, Wu D, Xu J. Calcitriol inhibits ROS-NLRP3-IL-1 $\beta$ signaling axis via activation of Nrf2-antioxidant signaling in hyperosmotic stress stimulated human corneal epithelial cells. Redox Biol. 2019 Feb; 21:101093.

34 Yu C, Chen P, Xu J, Liu Y, Li H, Wang L, et al. hADSCs derived extracellular vesicles inhibit NLRP3inflammasome activation and dry eye. Sci Rep. 2020 Sep;10(1):14521.
mADSC-Exos Inhibit NLRP3 Activation in Dry Eye
Ophthalmic Res 2022;65:40-51 DOI: $10.1159 / 000519458$ 